\title{
White Matter Changes Associated with Antipsychotic Treatment in First-Episode Psychosis
}

\author{
Philip R Szeszko*,1,2,3, Delbert G Robinson ${ }^{1,2,3}$, Toshikazu lkuta ${ }^{4}$, Bart D Peters ${ }^{1,2}$, Juan A Gallego ${ }^{1,2,3}$, \\ John Kane ${ }^{1,2,3}$ and Anil K Malhotra ${ }^{1,2,3}$ \\ 'Center for Psychiatric Neuroscience, The Feinstein Institute for Medical Research, Manhasset, NY, USA; ${ }^{2}$ Division of Psychiatry Research, Zucker \\ Hillside Hospital, North Shore-LII Health System, Glen Oaks, NY, USA; ${ }^{3}$ Departments of Psychiatry and Molecular Medicine, Hofstra North \\ Shore-LIJ School of Medicine, Hempstead, NY, USA; ${ }^{4}$ Department of Communication Sciences and Disorders, School of Applied Sciences, \\ University of Mississippi, University, MS, USA
}

Second-generation antipsychotics are utilized extensively in the treatment of psychotic disorders and other psychiatric conditions, but the effects of these medications on human brain white matter are not well understood. We thus investigated the effects of secondgeneration antipsychotics on white matter integrity using tract-based spatial statistics in patients experiencing a first episode of psychosis with little or no prior antipsychotic exposure, and how potential changes were associated with metabolic side effects. Thirty-five (26 men/9 women) patients experiencing a first episode of psychosis received diffusion tensor imaging (DTI) exams, clinical assessments, and provided fasting blood samples at the onset of antipsychotic treatment, and then again after 12 weeks of treatment with either risperidone or aripiprazole in a double-blind randomized clinical trial. In addition, 35 (26 men/9 women) healthy volunteers received DTI exams at a baseline time point and then after 12 weeks. Patients demonstrated significant $(p<0.05$; family-wise error corrected) fractional anisotropy reductions within the parietal and occipital white matter following antipsychotic treatment. Greater overall fractional anisotropy reduction was significantly correlated with greater increases in low-density lipoprotein. There were no significant fractional anisotropy increases among patients following treatment. Moreover, healthy volunteers did not demonstrate either significant increases or decreases in fractional anisotropy across a comparable 12-week interval. The use of antipsychotics may be associated with a subtle loss of white matter integrity that is related to greater side effects, thus raising potentially important considerations regarding risk/benefit in their usage. Limitations of the current study, however, include a prior history of substance use among patients and our inability to exclude the possibility of disease progression.

Neuropsychopharmacology (2014) 39, 1324-1331; doi:I0.1038/npp.2013.288; published online 19 February 2014

Keywords: diffusion tensor imaging; antipsychotic; first-episode psychosis; white matter

\section{INTRODUCTION}

Antipsychotic medications are widely prescribed not only for the treatment of schizophrenia and related psychotic disorders but also for a broad range of behavioral and/or disruptive disorders in children, adolescents, and the elderly. Thus, understanding the potential impact of antipsychotic medications on the brain has significant health implications. There are, however, limited longitudinal in-vivo data regarding the potential impact of antipsychotics on the brain, and, in particular, the white matter. Some studies suggest that antipsychotics may have an important therapeutic role in promoting myelination through their effects on oligodendrocytes (Walterfang et al, 2011; Bartzokis et al, 2011), with some empirical work

* Correspondence: Dr PR Szeszko, Division of Psychiatry Research, Zucker Hillside Hospital, North Shore-LII Health System, 75-59 263rd Street, Glen Oaks, NY I I004, USA, Tel: + I 718470 8489, Fax: + I 718343 1659, E-mail: szeszko@lij.edu

Received 3 June 2013; revised 27 August 2013; accepted 13 September 2013; accepted article preview online 16 October 2013 suggesting a protective or myelin-inducing effect of risperidone on the brain white matter (Bartzokis et al, 2007, 2011; Bartzokis et al, 2009). Similarly, animal studies noted that antipsychotic treatment following administration of the copper chelator, cuprizone, promoted oligodendrocyte development and remyelination (Xiao et al, 2008; Zhang et al, 2012), which was evident from magnetic resonance (MR) imaging and confirmed by histological measures (Chandran et al, 2012). In contrast to these studies, however, several longitudinal neuroimaging studies reported white matter volume decreases in association with greater exposure to antipsychotic medications (Ho et al, 2011) and white matter volume reductions in treatmentnaive patients following risperidone treatment (Molina et al, 2005; Girgis et al, 2006). Moreover, animal data report white matter volume reductions (Dorph-Petersen et al, 2005) and, to some extent, fewer oligodendrocytes (Konopaske et al, 2008) in macaques treated with olanzapine or haloperidol.

It is known that second-generation antipsychotics are associated with serious side effects that significantly increase risk for cardiometabolic disease (De Hert et al, 2011). In particular, second-generation antipsychotics have 
been linked to increased serum cholesterol and associated abnormalities in lipid metabolism (Hoffman et al, 2010; Bushe and Paton, 2005). However, the potential impact of antipsychotic-induced metabolic changes on the brain white matter has not been well investigated. This is particularly relevant given that $\sim 25 \%$ of the total amount of cholesterol in humans is localized to the brain, with the majority present in myelin (Björkhem and Meaney, 2004). Although most brain cholesterol is produced locally and the bloodbrain barrier protects it from exchange with lipoprotein cholesterol in the circulation, it is known that antipsychotics upregulate the expression of cholesterol transport proteins (Vik-Mo et al, 2009). Moreover, changes in cholesterol balance across the whole body can cause concomitant alterations in sterol recycling within the CNS that can alter the integrity of the myelin sheath (Dietschy and Turley, 2001). Consistent with this possibility are data from healthy humans, indicating that elevated serum cholesterol and, more specifically, greater low-density lipoprotein (LDL) is associated with lower white matter integrity among adults even below clinical thresholds for hyperlipidemia (Williams et al, 2012).

In the current study, we prospectively investigated the potential influence of antipsychotic medications on white matter integrity in patients experiencing a first episode of psychosis using longitudinal diffusion tensor imaging (DTI) to examine whether administration of antipsychotics would be associated with alterations in white matter integrity. Diffusion tensor-imaging exams were conducted in the context of a double-blind, randomized, controlled treatment trial comparing the efficacy and tolerability of risperidone and aripiprazole in patients experiencing a first episode of psychosis. Given prior work (Ho et al, 2011; Molina et al, 2005; Girgis et al, 2006), we hypothesized that antipsychotic treatment would be associated with reductions in FA, a putative measure of white matter integrity, in patients. Moreover, we examined the relationship between possible white matter changes and clinical response, as well as metabolic side effects associated with treatment.

\section{MATERIALS AND METHODS}

\section{Subjects}

Thirty-five patients experiencing a first episode of psychosis were recruited from admissions to the inpatient service at The Zucker Hillside Hospital in Glen Oaks, NY, and were enrolled in an NIMH-funded double-blind, randomized controlled trial comparing aripiprazole $v s$ risperidone (R01MH060004). All patients were required to have 2 weeks or less of cumulative lifetime exposure to antipsychotics to enter the clinical trial and received a physical exam and laboratory screening to rule out medical causes for their initial psychotic episode. All patient (lifetime) diagnoses were based on the SCID for Axis I DSM-IV Disorders supplemented by information from clinicians and, when available, family members. First-episode patients met DSMIV criteria for schizophrenia (undifferentiated $=15$ and paranoid $=9)$, schizophreniform disorder $(n=5)$, schizoaffective disorder $(N=1)$, or psychosis NOS $(N=5)$. Seventeen patients met criteria for a lifetime substance abuse/ dependence diagnosis, including (numbers in parentheses): alcohol dependence (3), alcohol abuse (3), amphetamine dependence (1), cannabis dependence (11), cannabis abuse (6), cocaine dependence (2), polysubstance dependence (1), and sedative, hypnotic, or anxiolytic abuse (1). However, in the month preceding the baseline and follow-up scans, 8 and 3 patients, respectively, met abuse/dependence criteria. On average, patients had a total lifetime exposure of 6.8 $(\mathrm{SD}=7.9)$ days of antipsychotic treatment before the baseline scan and were scanned, on average, within 3.1 $(\mathrm{SD}=7.7)$ days of entry into the clinical trial. One patient was treated with aripiprazole for $<2$ weeks before the baseline scan to meet study eligibility criteria for the clinical trial, but the exact number of days was unavailable. Twelve patients were antipsychotic drug naive at the time of the baseline scan. Mean age at first psychotic symptoms was 19.0 years $(\mathrm{SD}=3.0)$; data were unavailable for three patients.

In addition, we recruited 35 healthy volunteers from advertisements posted on websites to match the demographic distributions of patients. Exclusion criteria for healthy subjects included the presence of any lifetime history of a major mood or psychotic disorder as determined by clinical interview using the SCID-NP. Exclusion criteria for all study participants included MR imaging contraindications, neurologic conditions (Gilles de la Tourette's, Huntington's disease, Parkinson's disease, encephalitis, strokes, aneurysms, tumors, central nervous system infections, or degenerative brain diseases), any serious medical disorder that could affect brain functioning or mental retardation. This study was approved by the North Shore-Long Island Jewish Medical Center Institutional Review Board and written informed consent was obtained from all study participants or their parents in the case of minors. All minors provided written informed assent to participate in the study.

\section{Antipsychotic Titration Schedule}

Research psychiatrists followed a flexible-dosing titration schedule. The initial daily dose for patients in the treatment trial was $5 \mathrm{mg}$ for aripiprazole and $1 \mathrm{mg}$ for risperidone. The dose was initially increased after 3 days of treatment and then further adjustments were made every 1-3 weeks until the patient improved, developed side effects that precluded a dose increase, or reached a maximum daily dose of $30 \mathrm{mg}$ of aripiprazole or $6 \mathrm{mg}$ of risperidone. All patients were receiving either aripiprazole or risperidone at the time of the second scan, except one patient who discontinued treatment 2 weeks before the second scan. Lorazepam was prescribed for anxiety or agitation. Patients were not allowed to receive antidepressants or mood stabilizers, or any other psychotropic medication. Cogentin and/or propranolol was prescribed for extrapyramidal symptoms.

\section{Clinical and Metabolic Assessments}

Patients completed the 18-item Brief Psychiatric Rating Scale-Anchored version (BPRS-A; Overall and Gorham, 1962) and we derived a total score by summing all items. In addition, patients received the Schedule for Assessment of Negative Symptoms (SANS) Zucker Hillside Clinical Trials Version (Robinson et al, 2006), and the average of the global measures of affective flattening, avolition, alogia, and asociality was used as the outcome measure. Patients 
completed the clinical assessments, on average, within 3.6 $(\mathrm{SD}=7.7)$ and $4.6(\mathrm{SD}=7.0)$ days following the baseline and follow-up MR imaging exams, respectively. Average BPRS scores at the time of baseline scanning was 44.0 $(\mathrm{SD}=9.7)$ and $27.2(\mathrm{SD}=7.2)$ at follow-up. The average of the global ratings from the SANS was $2.1(\mathrm{SD}=0.8)$ at baseline and $1.8(\mathrm{SD}=0.6)$ at follow-up.

Blood samples (following all-night fasting) were drawn in the morning for assessment of cholesterol levels, including LDL and high-density lipoprotein (HDL). Patients completed these assessments, on average, within $1.4(\mathrm{SD}=8.2)$ and $4.2(\mathrm{SD}=7.7)$ days following the baseline and follow-up MR imaging exams, respectively. Total cholesterol at baseline was $159 \mathrm{mg} / \mathrm{dl}(\mathrm{SD}=24)$ and $174 \mathrm{mg} / \mathrm{dl}(\mathrm{SD}=30)$ at follow-up. LDL and HDL values at baseline were $91 \mathrm{mg} / \mathrm{dl}$ $(\mathrm{SD}=23)$ and $52 \mathrm{mg} / \mathrm{dl}(\mathrm{SD}=12)$, respectively, and at follow-up were $102 \mathrm{mg} / \mathrm{dl} \quad(\mathrm{SD}=24)$ and $52 \mathrm{mg} / \mathrm{dl}$ $(\mathrm{SD}=13)$, respectively. Complete data were unavailable for four patients.

\section{Handedness}

All individuals completed a modified version of the Edinburgh Inventory. The total number of right- and lefthand items was scored and the laterality quotient was computed according to the following formula: (Total $\mathrm{R}-$ Total $\mathrm{L}) /($ Total $\mathrm{R}+$ Total $\mathrm{L}$ ) yielding a range from +1.00 (totally dextral) to -1.00 (totally non-dextral).

\section{DTI Procedures}

MR imaging exams were conducted at the North Shore University Medical Center on a General Electric 3-T wholebody superconducting imaging system. All scans were reviewed by a radiologist for gross anatomic pathology that would preclude participation in this study. Scans were also reviewed by a member of the research team and scans with significant artifacts were repeated. A total of 36 DTI volumes were obtained from each subject that included 31 volumes with diffusion gradients applied along 31 nonparallel directions with $b=1000 \mathrm{~s} / \mathrm{mm}^{2}$ and 5 volumes without diffusion weighting $(b=0)$. Each volume consisted of 51 contiguous $2.5-\mathrm{mm}$ axial slices acquired parallel to the anterior-posterior commissural line using a ramp-sampled, spin-echo, single-shot echo-planar imaging method $(\mathrm{TR}=14000 \mathrm{~ms}, \mathrm{TE}=\mathrm{min}$, matrix $=128 \times 128, \mathrm{FOV}=240$ $\mathrm{mm})$.

\section{Image Processing and Tractography}

Image processing was conducted using TBSS (Smith et al, 2006) within the Functional Magnetic Resonance Imaging of the Brain Library (Jenkinson et al, 2012). Each subject's DTI data were aligned to the first $b=0$ volume, which was acquired without diffusion weighting, to correct eddycurrent distortion, and the $b$-vector table for each participant was adjusted accordingly. FA maps (radial diffusivity (RD) and axial diffusivity (AD) maps) were calculated for each voxel in the brain for all study participants using DTIFIT, part of FSL's diffusion toolbox. Each image was then non-linearly transformed using FSL's non-linear image registration tool into FMRIB58 FA standard brain for cross subject comparisons at the voxel level.

We used clusters that demonstrated FA reductions from the baseline to the follow-up time points among patients in the TBSS analysis as seed regions for tractography analysis (Behrens et al, 2007). Using the BEDPOSTX tool, the local (ie, within voxel) probability density functions of the principal diffusion direction were estimated using Markov chain Monte Carlo sampling; a spatial probability density function across voxels was then estimated based on these local probability density functions using the probtrackx tool. The parameters for PROBTRACKX included: number of samples $=5000, \quad$ curvature threshold $=0.2$, step length $=0.5 \mathrm{~mm}$, and number of steps per sample $=2000$. Tractography was conducted in native DTI space, and resultant pathways were linearly registered into FMRIB FA brain and averaged across all participants for visualization of the 'average' pathway(s) passing through the seed region(s).

\section{Statistical Analysis}

Group differences were assessed at baseline and follow-up using independent group Student's $t$-tests or $\chi^{2}$-tests, whereas longitudinal changes between the baseline and follow-up time points in each group were examined using paired Student's $t$-tests. We used permutation statistics (number of permutations $=5000$ ) via the Randomise tool in FSL with family-wise error correction $(p<0.05)$. We also employed threshold-free cluster enhancement in FSL (Smith and Nichols, 2009) to eliminate the need for arbitrary smoothing and cluster threshold criteria. Mean FA within significant clusters was exported to SPSS to examine in relation to clinical and metabolic measures (using a cutoff value of $p<0.05$; two-tailed) using either Pearson's product moment or Spearman's rank (in the case of non-normally distributed data) correlations.

\section{RESULTS}

The groups did not differ significantly in distributions of age, sex, handedness, smoking status, height, weight, education, or the number of weeks between the baseline and follow-up scans (Table 1). Total BPRS scores decreased significantly from the baseline to the follow-up MR imaging exam $(t=9.04, \mathrm{df}=31, p<0.001)$ but no significant changes in negative symptoms $(p>0.05)$ were evident. There were significant increases in cholesterol $(t=-3.13, \mathrm{df}=20$, $p=0.003)$ and LDL $(t=-2.81, \mathrm{df}=30, p=0.009)$ but no significant changes in HDL $(p>0.05)$ across the clinical trial. As expected, there were significant increases in body mass index from baseline to 12 weeks $(t=-7.07, \mathrm{df}=32$, $p<0.001)$.

Investigation of longitudinal changes using TBSS revealed seven regions within the white matter skeleton, where FA decreased significantly from baseline to the follow-up scan among patients (Figures 1 and 2). The size of these clusters ranged from 5 to 171 voxels (see Table 2) reflecting, on average, a $4 \%$ reduction in $\mathrm{FA}(\mathrm{SD}=2.5 \%$; range $=-0.1$ to $-9.5 \%)$ across these seven regions. Individual datapoints representing the average reduction in FA across the two 
Table I Baseline Sample Characteristics

\begin{tabular}{|c|c|c|c|c|c|}
\hline & First-episode patients $(N=35)$ & Healthy volunteers $(N=35)$ & df & Test statistic & $P$-value \\
\hline Age (years) & $21.5(4.6)$ & $21.8(4.8)$ & 68 & $t=0.26$ & NS \\
\hline $\operatorname{Sex}(M / F)$ & $26 \mathrm{M} / 9 \mathrm{~F}$ & $26 \mathrm{M} / 9 \mathrm{~F}$ & । & $\chi^{2}=0$ & NS \\
\hline Laterality quotient & $0.71(0.43)$ & $0.70(0.47)$ & 67 & $t=-0.04$ & NS \\
\hline Education (years) & $12.6(1.8)$ & $12.9(2.5)$ & 67 & $t=0.46$ & NS \\
\hline Height (inches) & $67.6(3.2)$ & $68.6(4.0)$ & 68 & $t=1.14$ & NS \\
\hline Scan interval (weeks) & $12.5(0.90)$ & $12.4(0.9 \mid)$ & 68 & $t=-0.19$ & NS \\
\hline
\end{tabular}

Notes: Data are presented as mean \pm SD in parentheses, unless otherwise indicated. There were data missing for the following variables: years of education ( 1 patient) and laterality quotient ( $\mathrm{l}$ patient).

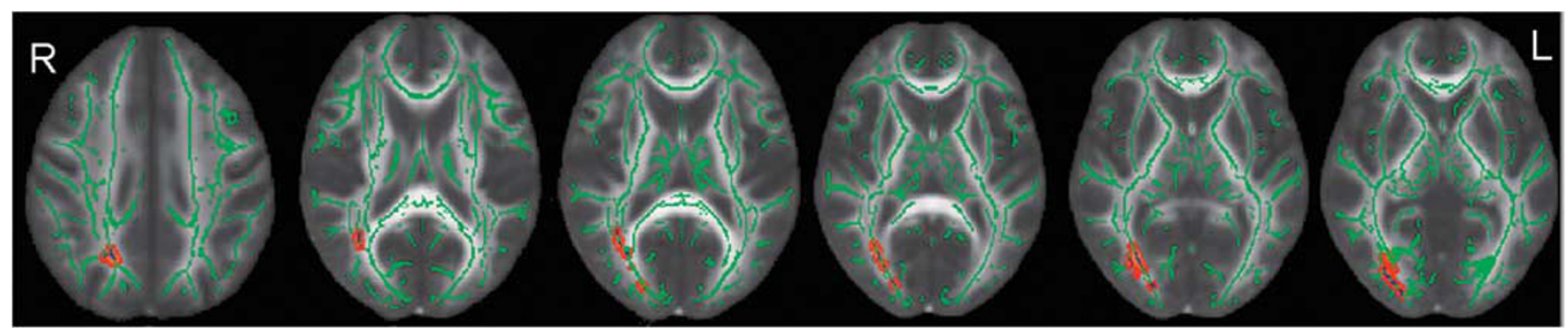

Figure I Significant fractional anisotropy reductions (in blue) Following 12 weeks of antipsychotic treatment in patients overlaid onto the TBSS skeleton. Note: for better visualization, significant clusters are 'thickened' (in red) using the 'TBSS_fill' script.'
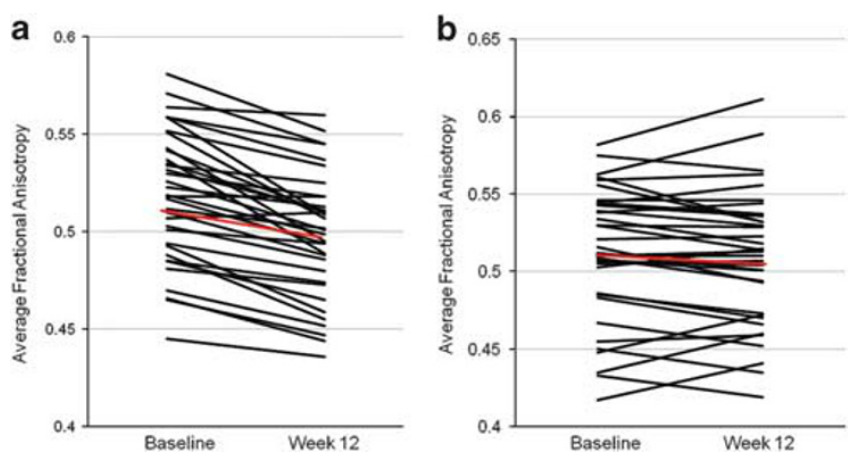

Figure 2 Individual changes in average fractional anisotropy across seven regions in patients (a) surviving family-wise error correction in TBSS from baseline to follow-up and corresponding changes within the average of these regions in healthy volunteers (b). Note: red line indicates mean change in both groups.

time points for patients and healthy volunteer individuals is provided in Figure 2. Additional analyses indicated that among patients, AD decreased $(t=4.32, \mathrm{df}=34, p<0.001)$ and $\mathrm{RD}$ increased $(t=-6.07, \mathrm{df}=34, p<0.001)$ across the average of these regions (individual regions are presented in Tables 2 and 3 for patients across the two time points). Significant $(p<0.05)$ changes in FA, RD, and $\mathrm{AD}$ across the average of the regions were evident among patients treated with either risperidone or aripiprazole. In contrast, among healthy volunteers there was, on average, a non-significant $-0.04 \%(\mathrm{SD}=3.3 \%)$ change in FA across the average of these seven regions (range $=-5.3$ to $6.3 \%$ ); moreover, no significant changes in $\mathrm{AD}$ or $\mathrm{RD}$ across these regions were evident among healthy volunteers $(p<0.05)$.

The single parietal and six occipital clusters were subsequently used as seed regions in probabilistic tractography. These analyses revealed that the parietal lobe cluster comprised the tapetum, traversing the splenium of the corpus callosum and extending toward the temporal lobe. Regions comprising the occipital clusters that differed across time points approximated posterior sections of the inferior longitudinal fasciculus/forceps major and possibly the inferior longitudinal fasciculus, and extended from the occipital region through the temporal lobe.

Given the lack of hypotheses regarding the correlates of changes in specific white matter regions and to minimize type-I error, we examined changes in clinical and metabolic measures in relationship to the average decrease in FA across the seven regions from the baseline to the follow-up scans. Greater reductions in FA correlated significantly with increases in LDL values $(r=-0.42, \mathrm{df}=29, p=0.019$; see Figure 3$)$ and, more specifically, $\mathrm{RD}(r=0.43, \mathrm{df}=29$, $p=0.016)$, but not $\mathrm{AD}(p>0.05)$. There was no significant correlation between changes in FA, RD, or AD and body mass index $(p>0.05)$. Changes in either positive or negative symptoms across the two time points did not correlate significantly with average change in FA across the time points. 
Table 2 Baseline and Follow-up Fractional Anisotropy Measures in Patients Surviving Family-wise Error Correction in TBSS

\begin{tabular}{ccccc}
\hline & Baseline & Follow-up & $\begin{array}{c}\text { FMRIB58 } \\
\text { coordinates } \\
(\boldsymbol{X}, \mathbf{Y}, \mathbf{Z})\end{array}$ & $\begin{array}{c}\text { Cluster } \\
\text { size }\end{array}$ \\
\hline $\begin{array}{c}\text { Parietal lobe } \\
\text { Cluster 1 }\end{array}$ & $0.568(0.064)$ & $0.545(0.061)$ & $20,-57,42$ & 64 \\
& & & & \\
Occipital lobe & & & & \\
Cluster 2 & $0.651(0.046)$ & $0.632(0.050)$ & $31,-68,5$ & 171 \\
Cluster 3 & $0.401(0.080)$ & $0.377(0.077)$ & $26,-83,1$ & 35 \\
Cluster 4 & $0.452(0.064)$ & $0.430(0.063)$ & $32,-78,1$ & 30 \\
Cluster 5 & $0.514(0.065)$ & $0.491(0.066)$ & $21,-85,11$ & 27 \\
Cluster 6 & $0.524(0.056)$ & $0.503(0.055)$ & $35,-70,5$ & 21 \\
Cluster 7 & $0.522(0.055)$ & $0.509(0.048)$ & $22,-85,5$ & 5 \\
\hline
\end{tabular}

Table 3 Baseline and Follow-up Axial and Radial Diffusivity Measures in Patients within Regions that Demonstrated Significant FA Reductions from Baseline to Follow-Up

\begin{tabular}{|c|c|c|c|c|c|}
\hline & Baseline & Follow-up & df & Test statistic & $P$-value \\
\hline \multicolumn{6}{|l|}{ Axial diffusivity } \\
\hline \multicolumn{6}{|l|}{ Parietal lobe } \\
\hline Cluster I & $1.288(0.082)$ & $1.267(0.081)$ & 34 & $t=3.03$ & 0.005 \\
\hline \multicolumn{6}{|l|}{ Occipital lobe } \\
\hline Cluster 2 & I.434 (0.082) & $1.412(0.073)$ & 34 & $t=3.11$ & 0.004 \\
\hline Cluster 3 & $1.086(0.077)$ & $1.076(0.070)$ & 34 & $t=1.22$ & NS \\
\hline Cluster 4 & $1.093(0.067)$ & $1.079(0.079)$ & 34 & $t=1.88$ & 0.069 \\
\hline Cluster 5 & $1.308(0.115)$ & $1.282(0.103)$ & 34 & $t=3.18$ & 0.003 \\
\hline Cluster 6 & I. $174(0.093)$ & I.I $56(0.083)$ & 34 & $t=2.27$ & 0.030 \\
\hline Cluster 7 & $1.283(0.118)$ & $1.279(0.113)$ & 34 & $t=0.43$ & 0.670 \\
\hline \multicolumn{6}{|l|}{ Radial diffusivity } \\
\hline \multicolumn{6}{|l|}{ Parietal lobe } \\
\hline Cluster I & $0.484(0.054)$ & $0.505(0.052)$ & 34 & $t=-5.12$ & $<0.001$ \\
\hline \multicolumn{6}{|l|}{ Occipital lobe } \\
\hline Cluster 2 & $0.430(0.047)$ & $0.446(0.05 I)$ & 34 & $t=-4.42$ & $<0.001$ \\
\hline Cluster 3 & $0.585(0.060)$ & $0.605(0.059)$ & 34 & $t=-4.55$ & $<0.001$ \\
\hline Cluster 4 & $0.531(0.047)$ & $0.548(0.050)$ & 34 & $t=-3.28$ & 0.002 \\
\hline Cluster 5 & $0.553(0.054)$ & $0.570(0.053)$ & 34 & $t=-4.13$ & $<0.001$ \\
\hline Cluster 6 & $0.495(0.047)$ & $0.509(0.047)$ & 34 & $t=-3.44$ & 0.002 \\
\hline Cluster 7 & $0.484(0.054)$ & $0.557(0.044)$ & 34 & $t=-2.12$ & 0.041 \\
\hline
\end{tabular}

Note: Units are $10^{-3} \mathrm{~mm}^{2} / \mathrm{s}$.

We investigated the potential effects of substance use on the observed findings. Patients with a lifetime substance use/dependence diagnosis demonstrated significant reductions in $\mathrm{FA}(t=6.00, \mathrm{df}=17, p<0.001)$ and $\mathrm{AD}(t=3.22$, $\mathrm{df}=17, p=0.005)$, as well as increases in $\mathrm{RD}(t=-3.45$, $\mathrm{df}=17, p=0.003$ ) across the two time points. Similarly, patients without a lifetime substance use/dependence diagnosis also demonstrated significant changes across the two time points with overall reductions in FA $(t=7.02$, $\mathrm{df}=16, p<0.001)$ and $\mathrm{AD}(t=2.80, \mathrm{df}=16, p=0.013)$, with increases in $\mathrm{RD}(t=-5.43, \mathrm{df}=16, p<0.001)$. Moreover, significant $(p<0.05)$ reductions in $\mathrm{FA}$ and $\mathrm{AD}$, as well as increases in $\mathrm{RD}$, were evident across the two time points in the subgroup of patients without any abuse or dependence in the month preceding the baseline scan (the only subgroup large enough for analysis).

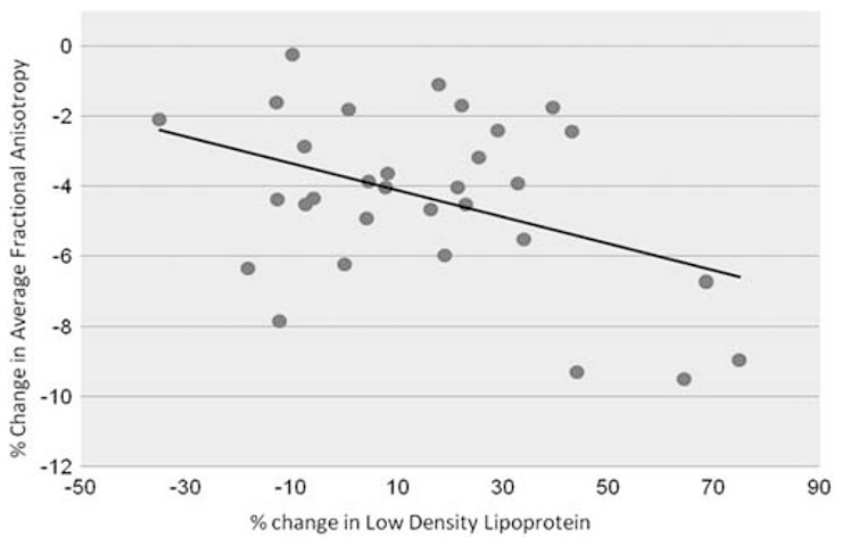

Figure 3 Scatterplot of changes in average fractional anisotropy and low-density lipoprotein in patients following 12 weeks of antipsychotic treatment.

\section{DISCUSSION}

Our DTI study, performed in the context of a double-blind, randomized, controlled treatment trial, provided a unique opportunity for clarifying the potential effects of antipsychotics on white matter integrity in patients experiencing a first episode of psychosis early in the course of illness and before extensive pharmacologic intervention. Our results suggest that reductions in FA, a putative measure of white matter integrity, are evident in parietal and occipital regions following $\sim 12$ weeks of atypical antipsychotic treatment. Study strengths include the use of a conservative tractinvariant analysis of FA data, family-wise error correction, limited treatment history of patients before baseline scanning, controlled antipsychotic treatment between scans, and the use of a healthy volunteer group well matched to patients for age, sex, and interscan interval. The lack of any significant FA changes among healthy volunteers who were scanned over the same time period supports the reliability of the DTI measurements.

Our findings are broadly consistent with prior work over a similar timeframe using structural imaging (Lieberman et al, 2005) and other longitudinal morphometric studies investigating white matter volume changes related to antipsychotic treatment. In one of the largest studies, involving a total of $674 \mathrm{MR}$ imaging scans and yielding an average of 3 scans per patient over the course of 7.2 years, Ho et al (2011) reported progressive white matter volume reductions among patients with schizophrenia, who received the greatest amount of antipsychotic treatment. Similarly, both Molina et al (2005) and Girgis et al (2006) reported white matter volume reductions in treatment-naive patients following 2 years and 6 weeks of treatment with risperidone, respectively. Notably, in these studies, white matter volume increases were not evident among patients and no white matter volume changes were observed in healthy volunteers. Moreover, our findings also converge with animal data, suggesting that administration of atypical antipsychotics is associated with changes in the brain gray and white matter in macaques treated with oral haloperidol or olanzapine compared with a sham group over a 17- to 27month period (Dorph-Petersen et al, 2005).

Little in-vivo research has been directed at understanding the relationship between antipsychotic treatment and 
changes in white matter integrity, as inferred by DTI imaging, using a longitudinal design. Our findings converge strongly, however, with those described by Wang et al (2013) who reported that compared with 22 healthy controls, there was a significant decrease in absolute FA in the white matter in first-episode drug-naive patients with schizophrenia after 6 weeks of antipsychotic treatment that did not correlate with symptom reduction. In our study, FA reductions were subtle and were most likely detected through the use of a within-subjects design, thus affording greater statistical power compared with crosssectional studies. In contrast, no FA increases were observed in patients over the 12 -week treatment period. It is conceivable that microstructural white matter changes may be related to metabolic changes associated with antipsychotic treatment, as such changes have been reported to occur among individuals with the metabolic syndrome (Shimoji et al, 2013). For example, recent work (Karlsson et al, 2013) suggests that obese individuals have lower FA in brain regions associated with reward seeking and appetite, and that FA was negatively associated with body fat percentage.

There may be several additional explanations regarding the observed FA reductions following antipsychotic treatment. Such reductions could be due to alterations in the composition of glial cells, which have been strongly implicated in several psychiatric disorders, including schizophrenia (Blank and Prinz, 2013). Furthermore, macaque data indicate that chronic antipsychotic treatment was associated with a reduction in glial cell numbers (Konopaske et al, 2008). Changes in white matter integrity in association with antipsychotic treatment may be related to specific alterations in macroglia and, in particular, oligodendrocytes, which form the myelin sheath, and together comprise the bulk of the white matter. In this regard, it is noteworthy that myelin comprises primarily of lipids, which have also been demonstrated to be affected by antipsychotic treatment (Huang and Chen, 2005). The finding of $\mathrm{AD}$ reductions and $\mathrm{RD}$ increases supports the possibility that axonal and myelin-related properties of the white matter (Song et al, 2002, 2003, 2005) may be altered by antipsychotic medications, and thereby contribute to the observed FA reductions. Moreover, changes in the expression of myelin basic protein, which have been linked to antipsychotic use (Kimoto et al, 2011), may have also contributed to the white matter changes observed herein.

In the current study, FA reductions in more posterior regions, but not in anterior regions, were associated with antipsychotic treatment. It is possible that longer exposure to antipsychotics could be associated with changes in more anterior regions. In addition, it is also conceivable that FA alterations might already be evident within frontal and temporal lobe regions among patients consistent with neurobiological models of schizophrenia (Szeszko et al, $2005,2008)$. It is noteworthy that a recent ultra-high field (7 T) combined DTI and magnetization transfer imaging study by Palaniyappan et al (2013) reported white matter abnormalities in occipito-temporal regions in 17 clinically stable patients with schizophrenia compared with that in 20 controls. These abnormalities were associated with impaired processing speed. Moreover, we reported previously that compared with patients with schizophrenia, who did not respond to antipsychotic treatment, those who did respond had more pronounced abnormalities in the occipital regions (Szeszko et al, 2012). Thus, we hypothesize that occipital FA changes may be a potentially important consideration in identifying treatment targets in schizophrenia.

Reductions in FA among patients were associated with increases in LDL across the treatment trial. Although there are limited data regarding the relationship between FA and metabolic measures, our findings converge strongly with cross-sectional data from Williams et al (2012) who reported that higher LDL was associated with lower FA among older healthy individuals below the clinical threshold for hyperlipidemia. Other data suggest that LDL levels are associated with greater severity of cerebral white matter changes on MR imaging (Crisby et al, 2010). Although metabolic changes are typically associated with antipsychotic treatment, the underlying neurobiology regarding concomitant brain changes is not well understood. Approximately one-quarter of the sterol within the body is localized to the brain (Dietschy and Turley, 2004) and is considered a major constituent of myelin such that cholesterol within oligodendrocytes has a key role in brain maturation (Saher et al, 2005). It is unlikely that changes in serum cholesterol directly impact metabolism of sterol within the brain, given that such an exchange does not occur across the blood-brain barrier (Björkhem and Meaney, 2004). For the maintenance of adequate brain cholesterol, the excess is excreted through cytochrome P-450-generated oxysterol 24S-hydroxycholesterol, thus permitting crossing of the blood-brain barrier (Björkhem and Meaney, 2004). Antipsychotics have been demonstrated to upregulate genes involved in cholesterol biosynthesis with subsequent changes in gene expression followed by enhanced HMGCR (3-hydroxy-3-methylglutaryl-coenzyme A reductase) enzyme activity and concomitant increases in cellular cholesterol (Ferno et al, 2005). Moreover, changes in the balance of cholesterol between the brain and other body compartments may be associated with concomitant alterations in sterol within the CNS, thereby affecting white matter integrity (Dietschy and Turley, 2001).

There were a number of limitations to our study and other possible explanations regarding the study findings that should be acknowledged. Although patients were treated for a relatively brief period of time, we cannot definitively rule out disease progression given the lack of an untreated patient group, and because patients are typically ill for 1-2 years before initiating treatment (Robinson et al, 2006). In addition, we did not investigate the potential effects of long-term antipsychotic treatment on the brain; however, our data are consistent with findings of lower FA throughout the white matter in DTI studies of chronically treated patients (Lim et al, 1999; Friedman et al, 2008). In addition, we did not collect cardiometabolic data in healthy volunteers and we acknowledge that smoking patterns, which have been reported to alter measures of white matter integrity (Gons et al, 2011; Paul et al, 2008), could conceivably affect the findings in patients reported herein, although the proportion of smokers in each group did not differ significantly. Lower FA could also be related to elevated body mass index (Xu et al, 2013; Stanek et al, 2011) and diabetes mellitus (Kodl et al, 2008); however, none of 
the subjects in our study had or developed diabetes during the treatment trial. A prior history of substance use among patients should also be noted as a possible limitation, although post-hoc analyses did not identify any effect of substance abuse/dependence on the observed FA reductions. Lastly, it should be acknowledged that several studies (Jones et al, 2005; Voineskos et al, 2010) reported that elderly patients with schizophrenia do not differ from healthy volunteers on some measures of white matter integrity, including those investigated in the current study. Thus, despite prior antipsychotic medication exposure, some patients may be resistant to the effects described in the current study.

In sum, we report reductions in white matter integrity after antipsychotic treatment in individuals experiencing a first episode of psychosis. Additional studies are needed to clarify the potential long-term effects of antipsychotics on white matter integrity and the identification of potential mechanisms to reverse such changes.

\section{FUNDING AND DISCLOSURE}

This work was supported in part by grants from NARSAD (PRS) and the National Institute of Mental Health to Dr Szeszko (R01 MH076995), Dr Robinson (R01 MH060004), the NSLIJ Research Institute General Clinical Research Center (M01 RR018535), an Advanced Center for Intervention and Services Research (P30 MH090590), and a Center for Intervention Development and Applied Research (P50 MH080173). JK is a consultant for Organon, Eli Lilly, BMS, Intracellular Therapeutics, Boehringer, Rules Based Medicine, Astra Zeneca, Otsuka, Novartis, Merck, Myriad, Esai, Pfizer, Lundbeck, J \& J, Targacept, Shire, Amgen, Sunovion, Pierre Fabre, Janssen, Alkermes, Jazz, and Forest Labs, and is on the Speakers Bureau for Janssen, Otsuka, BMS, Eli Lilly. AKM has had professional financial involvement with Genomind, Shire, Eli Lilly, Sunovion, Abbott, and in income sources/equity of $\$ 10000$ per year or greater in Genomind and grant funding from Abbott. DR is a consultant for Asubio Pharmaceuticals and Shire, and has received grant support from Bristol Myers Squibb and Janssen. PRS, TI, $\mathrm{BDP}$, and JAG declare no conflicts of interest.

\section{REFERENCES}

Bartzokis G, Lu PH, Amar CP, Raven EP, Detore NR, Altshuler LL et al (2011). Long acting injection $v s$ oral risperidone in firstepisode schizophrenia: differential impact on white matter myelination trajectory. Schizophr Res 132: 35-41.

Bartzokis G, Lu PH, Nuechterlein KH, Gitlin M, Doi C, Edwards N et al (2007). Differential effects of typical and atypical antipsychotics on brain myelination in schizophrenia. Schizophr Res 93: 13-22.

Bartzokis G, Lu PH, Stewart SB, Oluwadara B, Lucas AJ, Pantages J et al (2009). In vivo evidence of differential impact of typical and atypical antipsychotics on intracortical myelin in adults with schizophrenia. Schizophr Res 113: 322-331.

Behrens TE, Berg HJ, Jbabdi S, Rushworth MF, Woolrich MW (2007). Probabilistic diffusion tractography with multiple fibre orientations: What can we gain? Neuroimage 34: 144-155.

Björkhem I, Meaney S (2004). Brain cholesterol: long secret life behind a barrier. Arterioscler Thromb Vasc Biol 24: 806-815.
Blank T, Prinz M (2013). Microglia as modulators of cognition and neuropsychiatric disorders. Glia 61: 62-70.

Bushe C, Paton C (2005). The potential impact of antipsychotics on lipids in schizophrenia: is there enough evidence to confirm a link? J Psychopharmacol 19: 76-83.

Chandran P, Upadhyay J, Markosyan S, Lisowski A, Buck W, Chin $\mathrm{CL}$ et al (2012). Magnetic resonance imaging and histological evidence for the blockade of cuprizone-induced demyelination in C57BL/6 mice. Neuroscience 202: 446-453.

Crisby M, Bronge L, Wahlund LO (2010). Low levels of high density lipoprotein increase the severity of cerebral white matter changes: implications for prevention and treatment of cerebrovascular diseases. Curr Alzheimer Res 7: 534-539.

De Hert M, Detraux J, van Winkel R, Yu W, Correll CU (2011). Metabolic and cardiovascular adverse effects associated with antipsychotic drugs. Nat Rev Endocrinol 8: 114-126.

Dietschy JM, Turley SD (2001). Cholesterol metabolism in the brain. Curr Opin Lipidol 12: 105-112.

Dietschy JM, Turley SD (2004). Thematic review series: brain lipids. Cholesterol metabolism in the central nervous system during early development and in the mature animal. J Lipid Res 45: 1375-1397.

Dorph-Petersen KA, Pierri JN, Perel JM, Sun Z, Sampson AR, Lewis DA (2005). The influence of chronic exposure to antipsychotic medications on brain size before and after tissue fixation: a comparison of haloperidol and olanzapine in macaque monkeys. Neuropsychopharmacology 30: 1649-1661.

Ferno J, Raeder MB, Vik-Mo AO, Skrede S, Glambek M, Tronstad KJ et al (2005). Antipsychotic drugs activate SREBP-regulated expression of lipid biosynthetic genes in cultured human glioma cells: a novel mechanism of action? Pharmacogenomics J 5: 298-304.

Friedman JI, Tang C, Carpenter D, Buchsbaum M, Schmeidler J, Flanagan L et al (2008). Diffusion tensor imaging findings in first-episode and chronic schizophrenia patients. Am J Psychiatry 165: 1024-1032.

Girgis RR, Diwadkar VA, Nutche JJ, Sweeney JA, Keshavan MS, Hardan AY (2006). Risperidone in first-episode psychosis: a longitudinal, exploratory voxel-based morphometric study. Schizophr Res 82: 89-94.

Gons RA, van Norden AG, de Laat KF, van Oudheusden LJ, van Uden IW, Zwiers MP et al (2011). Cigarette smoking is associated with reduced microstructural integrity of cerebral white matter. Brain 134: 2116-2124.

Ho BC, Andreasen NC, Ziebell S, Pierson R, Magnotta V (2011). Long-term antipsychotic treatment and brain volumes: a longitudinal study of first-episode schizophrenia. Arch Gen Psychiatry 68: 128-137.

Hoffmann VP, Case M, Stauffer VL, Jacobson JG, Conley RR (2010). Predictive value of early changes in triglycerides and weight for longer-term changes in metabolic measures during olanzapine, ziprasidone or aripiprazole treatment for schizophrenia and schizoaffective disorder post hoc analyses of 3 randomized, controlled clinical trials. J Clin Psychopharmacol 30: 656-660.

Huang TL, Chen JF (2005). Serum lipid profiles and schizophrenia: effects of conventional or atypical antipsychotic drugs in Taiwan. Schizophr Res 80: 55-59.

Jenkinson M, Beckmann CF, Behrens TE, Woolrich MW, Smith SM (2012). FSL. NeuroImage 62: 782-790.

Jones DK, Catani M, Pierpaoli C, Reeves SJ, Shergill SS, O’Sullivan $M$ et al (2005). A diffusion tensor magnetic resonance imaging study of frontal cortex connections in very-late-onset schizophrenia-like psychosis. Am J Geriatr Psychiatry 13: 1092-1099 PubMed PMID: 16319302.

Karlsson HK, Tuulari JJ, Hirvonen J, Lepomäki V, Parkkola R, Hiltunen J et al (2013). Obesity is associated with white matter atrophy: A combined diffusion tensor imaging and voxel-based morphometric study. Obesity (Silver Spring) 21: 2530-2537. 
Kimoto S, Okuda A, Toritsuka M, Yamauchi T, Makinodan M, Okuda $\mathrm{H}$ et al (2011). Olanzapine stimulates proliferation but inhibits differentiation in rat oligodendrocyte precursor cell cultures. Prog Neuropsychopharmacol Biol Psychiatry 35: 1950-1956.

Kodl CT, Franc DT, Rao JP, Anderson FS, Thomas W, Mueller BA et al (2008). Diffusion tensor imaging identifies deficits in white matter microstructure in subjects with type 1 diabetes that correlate with reduced neurocognitive function. Diabetes 57: 3083-3089.

Konopaske GT, Dorph-Petersen KA, Sweet RA, Pierri JN, Zhang W, Sampson AR et al (2008). Effect of chronic antipsychotic exposure on astrocyte and oligodendrocyte numbers in macaque monkeys. Biol Psychiatry 63: 759-765.

Lieberman JA, Tollefson GD, Charles C, Zipursky R, Sharma T, Kahn RS et al (2005). Antipsychotic drug effects on brain morphology in first-episode psychosis. Arch Gen Psychiatry 62: 361-370.

Lim KO, Hedehus M, Moseley M, de Crespigny A, Sullivan EV, Pfefferbaum A (1999). Compromised white matter tract integrity in schizophrenia inferred from diffusion tensor imaging. Arch Gen Psychiatry 56: 367-374.

Molina V, Reig S, Sanz J, Palomo T, Benito C, Sanchez J et al (2005). Increase in gray matter and decrease in white matter volumes in the cortex during treatment with atypical neuroleptics in schizophrenia. Schizophr Res 80: 61-71.

Overall JE, Gorham DR (1962). The brief psychiatric rating scale. Psychol Rep 10: 799-812.

Palaniyappan L, Al-Radaideh A, Mougin O, Gowland P, Liddle PF (2013). Combined white matter imaging suggests myelination defects in visual processing regions in schizophrenia. Neuropsychopharmacology 38: 1808-1815.

Paul RH, Grieve SM, Niaura R, David SP, Laidlaw DH, Cohen R et al (2008). Chronic cigarette smoking and the microstructural integrity of white matter in healthy adults: a diffusion tensor imaging study. Nicotine Tob Res 10: 137-147.

Robinson DG, Woerner MG, Napolitano B, Patel RC, Sevy SM, Gunduz-Bruce $\mathrm{H}$ et al (2006). Randomized comparison of olanzapine versus risperidone for the treatment of first-episode schizophrenia: 4-month outcomes. Am J Psychiatry 163: 20962102.

Saher G, Brügger B, Lappe-Siefke C, Möbius W, Tozawa R, Wehr MC et al (2005). High cholesterol level is essential for myelin membrane growth. Nat Neurosci 8: 468-475.

Shimoji K, Abe O, Uka T, Yasmin H, Kamagata K, Asahi K et al (2013). White matter alteration in metabolic syndrome: diffusion tensor analysis. Diabetes Care 36: 696-700.

Smith SM, Jenkinson M, Johansen-Berg H, Rueckert D, Nichols TE, Mackay CE et al (2006). Tract-based spatial statistics: voxelwise analysis of multi-subject diffusion data. Neuroimage 31: 1487-1505.

Smith SM, Nichols TE (2009). Threshold-free cluster enhancement: Addressing problems of smoothing, threshold dependence and localisation in cluster inference. Neuroimage 44: 83-98.

Song SK, Sun SW, Ju WK, Lin SJ, Cross AH, Neufeld AH (2003). Diffusion tensor imaging detects and differentiates axon and myelin degeneration in mouse optic nerve after retinal ischemia. Neuroimage 20: 1714-1722.

Song SK, Sun SW, Ramsbottom MJ, Chang C, Russell J, Cross AH (2002). Dysmyelination revealed through MRI as increased radial (but unchanged axial) diffusion of water. Neuroimage 17: 1429-1436.

Song SK, Yoshino J, Le TQ, Lin SJ, Sun SW, Cross AH et al (2005). Demyelination increases radial diffusivity in corpus callosum of mouse brain. Neuroimage 26: 132-140.

Stanek KM, Grieve SM, Brickman AM, Korgaonkar MS, Paul RH, Cohen RA et al (2011). Obesity is associated with reduced white matter integrity in otherwise healthy adults. Obesity (Silver Spring) 19: 500-504.

Szeszko PR, Narr KL, Phillips OR, McCormack J, Sevy S, GunduzBruce $\mathrm{H}$ et al (2012). Magnetic resonance imaging predictors of treatment response in first episode schizophrenia. Schizophr Bull 38: $569-578$.

Szeszko PR, Ardekani BA, Ashtari M, Kumra S, Robinson DG, Sevy $S$ et al (2005). White matter abnormalities in first-episode schizophrenia or schizoaffective disorder: a diffusion tensor imaging study. Am J Psychiatry 162: 602-605.

Szeszko PR, Robinson DG, Ashtari M, Vogel J, Betensky J, Sevy S et al (2008). Clinical and neuropsychological correlates of white matter abnormalities in recent onset schizophrenia. Neuropsychopharmacology 33: 976-984.

Vik-Mo AO, Ferno J, Skrede S, Steen VM (2009). Psychotropic drugs up-regulate the expression of cholesterol transport proteins including ApoE in cultured human CNS- and liver cells. BMC Pharmacol 9: 10.

Voineskos AN, Lobaugh NJ, Bouix S, Rajji TK, Miranda D, Kennedy JL et al (2010). Diffusion tensor tractography findings in schizophrenia acrosss the adult lifespan. Brain 133: 1494-1504.

Walterfang M, Velakoulis D, Whitford TJ, Pantelis C (2011). Understanding aberrant white matter development in schizophrenia: an avenue for therapy? Expert Rev Neurother 11: 971-987.

Wang Q, Cheung C, Deng W, Li M, Huang C, Ma X et al (2013). White-matter microstructure in previously drug naive patients with schizophrenia after 6 weeks of treatment. Psychol Med 43: 1-9.

Williams VJ, Leritz EC, Shepel J, McGlinchey RE, Milberg WP, Rudolph JL et al (2012). Interindividual variation in serum cholesterol is associated with regional white matter tissue integrity in older adults. Hum Brain Mapp 34: 1826-1841.

Xiao L, Xu H, Zhang Y, Wei Z, He J, Jiang W et al (2008). Quetiapine facilitates oligodendrocyte development and prevents mice from myelin breakdown and behavioral changes. $\mathrm{Mol}$ Psychiatry 13: 697-708.

Xu J, Li Y, Lin H, Sinha R, Potenza MN (2013). Body mass index correlates negatively with white matter integrity in the fornix and corpus callosum: a diffusion tensor imaging study. Hum Brain Mapp 34: 1044-1052.

Zhang Y, Zhang H, Wang L, Jiang W, Xu H, Xiao L et al (2012). Quetiapine enhances oligodendrocyte regeneration and myelin repair after cuprizone-induced demyelination. Schizophr Res 138: $8-17$. 